PROCEEDINGS OF THE

AMERICAN MATHEMATICAL SOCIETY

Volume 127, Number 12, Pages 3533-3545

S 0002-9939(99)05379-4

Article electronically published on August 5, 1999

\title{
ON X-RAY TRANSFORMS FOR RIGID LINE COMPLEXES AND INTEGRALS OVER CURVES IN $\mathbb{R}^{4}$
}

\author{
ALLAN GREENLEAF, ANDREAS SEEGER, AND STEPHEN WAINGER \\ (Communicated by Christopher D. Sogge)
}

\begin{abstract}
Endpoint estimates are proved for model cases of restricted X-ray transforms and singular fractional integral operators in $\mathbb{R}^{4}$.
\end{abstract}

\section{INTRODUCTION}

Let $G_{1, d}$ be the Grassmannian of all lines in $\mathbb{R}^{d}$ passing through the origin. Let $H$ be a hyperplane in $\mathbb{R}^{d}$ and let $\Gamma$ be a compact one-dimensional submanifold of $G_{1, d}$ consisting of lines which do not lie in $H$. Let $\mathfrak{C}$ be the $d$-dimensional line complex of all lines of the form $x^{\prime}+\ell$, where $x^{\prime} \in H$ and $\ell \in \Gamma$. We refer to $\mathfrak{C}$ as a rigid line complex; it serves as a model case for more general classes of line complexes, considered in [5], [8], [6], [7].

The restricted $\mathrm{X}$-ray transform associated to $\mathfrak{C}$ is defined by

$$
R_{\mathfrak{C}} f\left(x^{\prime}, \ell\right)=\int_{x^{\prime}+\ell} f d s, \quad \ell \in \Gamma, x^{\prime} \in H .
$$

We shall consider line complexes satisfying a curvature assumption. If $\Gamma$ is parametrized by $\alpha \rightarrow \Gamma(\alpha)$, then $\mathfrak{C}$ is defined to be well-curved if $\Gamma^{\prime}(\alpha), \Gamma^{\prime \prime}(\alpha), \ldots, \Gamma^{(d-1)}(\alpha)$ are linearly independent. Note that this well-curvedness assumption does not depend on the specific parametrization of $\Gamma$.

We are interested in the $L^{p}\left(\mathbb{R}^{d}\right) \rightarrow L^{q}(\mathfrak{C})$ mapping properties of the X-ray transform; in defining the space $L^{q}(\mathfrak{C})$ we use the measure $d x^{\prime} d \sigma$ on $\mathfrak{C}=H \times \Gamma$, where $d x^{\prime}$ is Lebesgue measure on $H$ and $d \sigma$ may be any positive smooth density on $\Gamma$. Homogeneity considerations show that the relation $d / p-(d-1) / q=1$ is necessary for $L^{p} \rightarrow L^{q}$ boundedness. Assuming this restriction it is conjectured that $L^{p} \rightarrow L^{q}$ boundedness holds for $1 \leq p \leq d(d+1) /\left(d^{2}-d+2\right)$. The latter restriction on $p$ is necessary, as can be seen by testing $R$ on characteristic functions of rectangles with dimensions $\left(\delta, \delta^{2}, \ldots, \delta^{d-1}, \delta\right)$ (see $\left.[7]\right)$. The conjecture has been proved for not necessarily rigid line complexes in $\mathbb{R}^{3}$ (see [6]); moreover partial results in higher dimensions, in the rigid case for some local versions, have been obtained in [7], for the range $p \geq 2\left(d^{2}-d\right) /\left(d^{2}-d+2\right)$. During the final stage of preparation of this paper we received the preprint [16] by Oberlin where another partial result, namely a restricted weak type estimate for $p=d /(d-1), q=(d-1) /(d-2)$, is proved.

Received by the editors January 13, 1998.

1991 Mathematics Subject Classification. Primary 44A12; Secondary 35S30.

This research was supported in part by grants from the National Science Foundation.

(C)1999 American Mathematical Society 
Theorem 1.1. Let $\mathfrak{C}=H \times \Gamma$ be a rigid well-curved line complex in $\mathbb{R}^{4}$. Then the operator $R_{\mathfrak{C}}$ is bounded from $L^{p}\left(\mathbb{R}^{4}\right)$ to $L^{q}(\mathfrak{C})$ if $4 / p-3 / q=1$ and $1 \leq p<10 / 7$. Moreover $R_{\mathfrak{C}}$ is of restricted weak type $(10 / 7,5 / 3)$, i.e. it maps the space $L^{10 / 7,1}\left(\mathbb{R}^{4}\right)$ to the space $L^{5 / 3, \infty}(\mathfrak{C})$.

Here $L^{p, q}$ is the familiar Lorentz space (see, e.g., [1], [19]). A consequence of Theorem 1.1 is of course that $R_{\mathfrak{C}}$ maps $L_{\text {comp }}^{p}$ into $L_{\text {loc }}^{q}$ if and only if $(1 / p, 1 / q)$ belongs to the triangle with vertices $(0,0),(1,1)$ and $(10 / 7,5 / 3)$, with the possible exception of the point $(10 / 7,5 / 3)$.

We shall also consider a model case of translation invariant operators, namely integrals along the curve

$$
\Gamma_{d}(t)=\left(t, t^{2}, \ldots, t^{d}\right)
$$

For any Schwartz-function $f$ on $\mathbb{R}^{d}$ and $0<\beta \leq 1$ define

$$
\mathcal{A}_{\beta} f(x)=\int_{-\infty}^{\infty} f\left(x-\Gamma_{d}(t)\right) t^{\beta-1} d t,
$$

where the case $\beta=1$ is of particular interest. Homogeneity considerations, with respect to the dilations $\delta \rightarrow\left(\delta, \delta^{2}, \ldots, \delta^{d}\right)$, show that the relation

$$
\frac{1}{p}-\frac{1}{q}=\frac{2 \beta}{d(d+1)}
$$

is necessary for $L^{p}\left(\mathbb{R}^{d}\right) \rightarrow L^{q}\left(\mathbb{R}^{d}\right)$ boundedness. It is conjectured that $\mathcal{A}_{\beta}$ maps $L^{p}$ to $L^{q}$ if and only if $(1.3)$ is satisfied and $(1 / p, 1 / q)$ belongs to the closed trapezoid $\mathcal{T}_{d}=\operatorname{hull}\left\{A, B, C_{d}, D_{d}\right\}$ where $A=(0,0), B=(1,1), C_{d}=\left(\frac{d^{2}-d+2}{d^{2}+d}, \frac{d-1}{d+1}\right), D_{d}=$ $\left(\frac{2}{d+1}, \frac{2 d-2)}{d^{2}+d}\right)$. Note that $C_{d}=D_{d} \Longleftrightarrow d=2$.

M. Christ [4] found an argument (partially based on ideas in [12]) showing how $L^{p} \rightarrow L^{q}$ estimates for the operator $\mathcal{A}_{\beta}$ can be deduced from a local version, namely

$$
T_{0} f(x)=\int_{1 \leq|t| \leq 2} f\left(x-\Gamma_{d}(t)\right) d t .
$$

It is conjectured that $T_{0}$ maps $L^{p} \rightarrow L^{q}$ if and only if $(1 / p, 1 / q) \in \mathcal{T}_{d}$. The necessity of this condition is known and attributed to A. Carbery and M. Christ in [10]; one tests $A$ on small balls and on small rectangles with dimensions $\delta \times \cdots \times \delta^{d}$. The conjecture is known in dimensions $d=2,3$; see Strichartz [20], Littman [9] for the case $d=2$, and Oberlin [13] for the case $d=3$. The latter case is accessible by $T^{*} T$ arguments because of the critical exponent $q=2$ for the vertex $C_{3}$ of $\mathcal{T}_{3}$. Based on Oberlin's result and the method in [4], S. Secco [18] established the conjectured bounds for $\mathcal{A}_{\beta}$ in three dimensions. Recently, Oberlin [15] showed that in four dimensions $T_{0}$ maps $L^{p} \rightarrow L^{q}$ whenever $(1 / p, 1 / q)$ belongs to the interior of $\mathcal{T}_{4}$, or to the line joining $E_{4}=(1 / 2,2 / 5)$ and $F_{4}=(3 / 5,1 / 2)$, a proper closed subset of the edge $\left(C_{4}, D_{4}\right)$. Combining this result with the method in [4] yields that the operator $\mathcal{A}_{\beta}$ is $L^{p} \rightarrow L^{q}$ bounded if $1 / p-1 / q=\beta / 10$ and if $\frac{10}{10-3 \beta}<p<\frac{5}{2 \beta}$, $0<\beta<1$, or $5 / 3 \leq p \leq 2, \beta=1$. $^{1}$

\footnotetext{
${ }^{1}$ After this paper had been submitted the authors received a preprint by Michael Christ entitled "Convolution, Curvature and Combinatorics - A Case Study" in which he shows by different methods that the operator $T_{0}$ is of restricted weak type at the endpoints $C_{d}, D_{d}$. This result, in conjunction with Proposition 4.1 can be used to prove the analogue of Theorem 1.2 in higher dimensions.
} 
Theorem 1.2. Suppose $d=4$ and $\mathcal{A}_{\beta}$ is as in (1.2).

(i) Let $0<\beta<1$. Then $\mathcal{A}_{\beta}$ maps $L^{p}\left(\mathbb{R}^{4}\right)$ boundedly to $L^{q}\left(\mathbb{R}^{4}\right)$ if and only if $1 / p-1 / q=\beta / 10$ and $\frac{10}{10-3 \beta} \leq p \leq \frac{5}{2 \beta}$; in fact $\mathcal{A}_{\beta}$ then maps $L^{p}\left(\mathbb{R}^{4}\right)$ to $L^{q, p}\left(\mathbb{R}^{4}\right)$.

(ii) Let $\beta=1$. The operator $\mathcal{A}_{1}$ is of restricted weak type $(10 / 7,5 / 3)$ and of restricted weak type $(5 / 2,10 / 3)$. It maps $L^{p}\left(\mathbb{R}^{4}\right)$ boundedly to $L^{q, p}\left(\mathbb{R}^{4}\right)$ if $1 / p-$ $1 / q=1 / 10$ and $10 / 7<p<5 / 2$.

For $(1 / p, 1 / q)$ in any open line segment of the boundedness set, the $L^{p} \rightarrow L^{q}$ estimate implies the sharper $L^{p} \rightarrow L^{q, p} \subset L^{q}$ estimate by real interpolation.

We use the following notation. $A \lesssim B$ means that there is a constant $C$ depending only on the underlying geometry such that $A \leq C B$. $L^{p}$ norms will be denoted by $\|\cdot\|_{p}$ and $L^{q, r}$ norms will be denoted by $\|\cdot\|_{q, r}$.

\section{RESTRICTED X-RAY TRANSFORMS}

In this section we give a proof of Theorem 1.1. By a change of variable we may assume that $H=\left\{\left(x^{\prime}, 0\right): x^{\prime} \in \mathbb{R}^{3}\right\}$. The assumption of rigidity means that the set $\mathfrak{C}_{x^{\prime}}$ is a translate of a fixed $\mathfrak{C}_{0} ; \mathfrak{C}_{x^{\prime}}$ is given as a family of lines $\ell_{x^{\prime}, \alpha}$ where $\ell_{x^{\prime}, \alpha}=\left\{\left(x^{\prime}+t \gamma(\alpha), t\right): t \in \mathbb{R}\right\}$; here $\alpha$ is restricted to a compact interval $I$ and $\gamma: I \rightarrow \mathbb{R}^{3}$ satisfies

$$
\Delta(\alpha):=\operatorname{det}\left(\gamma^{\prime}(\alpha) \quad \gamma^{\prime \prime}(\alpha) \quad \gamma^{\prime \prime \prime}(\alpha)\right) \neq 0 .
$$

Without loss of generality we may assume that $\gamma$ is a parametrization by arclength, i.e. $\left|\gamma^{\prime}(\alpha)\right|=1$.

We consider the operator

$$
R f\left(x^{\prime}, \alpha\right)=\chi(\alpha) \int_{-\infty}^{\infty} f\left(x^{\prime}-t \gamma(\alpha), t\right) d t
$$

where $\chi$ is a nonnegative smooth function supported in the interior of $I$. In view of the compactness of $\Gamma$ the $L^{p} \rightarrow L^{q}$ estimates for $R$ immediately imply the same $L^{p} \rightarrow L^{q}$ estimates for $R_{\mathfrak{C}}$ as defined in (1.1). We shall show that the adjoint $R^{*}$ maps $L^{5 / 2,1}\left(\mathbb{R}^{3} \times \Gamma\right)$ to $L^{10 / 3, \infty}\left(\mathbb{R}^{4}\right)$; here

$$
R^{*} f\left(x^{\prime}, t\right)=\int f\left(x^{\prime}+t \gamma(\alpha), \alpha\right) \chi(\alpha) d \alpha
$$

and we may assume that $\chi$ has small support in $I$. The full result of Theorem 1.1 follows by duality and interpolation.

Let $\eta$ be an even Schwartz function on the real line with the property that $\hat{\eta}(\tau)=1$ for $|\tau| \leq 1 / 2$ and $\hat{\eta}(\tau)=0$ for $|\tau| \geq 1$. For $L \in \mathbb{Z}$ define

$$
B_{L} f\left(x^{\prime}, t\right)=\iint f\left(x^{\prime}+t \gamma(\alpha)+u \gamma^{\prime}(\alpha), \alpha\right) \chi(\alpha) 2^{L} \eta\left(2^{L} u\right) d \alpha d u ;
$$

note that $\lim _{L \rightarrow \infty} B_{L}=R^{*} f$. Our main estimates are

Proposition 2.1. For all $L \in \mathbb{Z}$

$$
\left\|R^{*} f-B_{L} f\right\|_{L^{2}\left(\mathbb{R}^{4}\right)} \lesssim 2^{-L / 2}\|f\|_{L^{2}\left(\mathbb{R}^{3} \times \Gamma\right)} .
$$

Proposition 2.2. For all $L \in \mathbb{Z}$

$$
\left\|B_{L} f\right\|_{L^{6}\left(\mathbb{R}^{4}\right)} \lesssim 2^{L / 3}\|f\|_{L^{3}\left(\mathbb{R}^{3} \times \Gamma\right)} .
$$


The idea of using an $L^{3} \rightarrow L^{6}$ estimate for averages over two-dimensional surfaces is due to Oberlin, and the proof of Proposition 2.2 will follow an analogous argument in [15, II], using multilinear operators. Given Propositions 2.1 and 2.2 one can use an argument by Bourgain [2] to complete the

Proof of Theorem 1.1. Let $E$ be a measurable set of finite measure in $\mathbb{R}^{3} \times \Gamma$. Choose $L$ such that $2^{-L} \leq\left(|E| \lambda^{-4}\right)^{1 / 3}<2^{-L+1}$. Then by Tshebyshev's inequality and Propositions 2.1 and 2.2

$$
\begin{aligned}
& \left|\left\{x:\left|R^{*} \chi_{E}(x)\right|>\lambda\right\}\right| \leq \frac{\left\|B_{L} \chi_{E}\right\|_{6}^{6}}{(\lambda / 2)^{6}}+\frac{\left\|R^{*} \chi_{E}-B_{L} \chi_{E}\right\|_{2}^{2}}{(\lambda / 2)^{2}} \\
& \quad \leq C\left(2^{2 L}|E|^{2} \lambda^{-6}+2^{-L} \lambda^{-2}|E|\right) \leq C^{\prime}|E|^{4 / 3} \lambda^{-10 / 3}=C^{\prime}\left\|\chi_{E}\right\|_{5 / 2}^{10 / 3} \lambda^{-10 / 3}
\end{aligned}
$$

This is saying that $R^{*}$ is of restricted weak type $(5 / 2,10 / 3)$ which by duality implies the assertion.

Proof of Proposition 2.1. As pointed out above we may assume that $\chi$ is supported in the interior of a small interval $I_{0} \subset I$. Let $\mathcal{B}_{\ell}=B_{\ell+1}-B_{\ell}$; since $R_{\mathfrak{C}}^{*} f-B_{L} f=$ $\sum_{\ell=L}^{\infty} \mathcal{B}_{\ell} f$ for Schwartz-functions $f$ it is sufficient to show that $\mathcal{B}_{\ell}^{*}$ is bounded on $L^{2}$ with norm $O\left(2^{-\ell / 2}\right)$.

Following [11] we apply the partial Fourier transform of $f$ with respect to the $x^{\prime}$ variables, denoted here by $\widetilde{f}$. Then define $\zeta$ by $\widehat{\zeta}(\tau)=\eta(\tau)-2 \eta(2 \tau) ; \zeta$ is even and supported in $\pm[1 / 2,2]$. Then

$$
\widetilde{\mathcal{B}_{\ell}^{*} f}(\xi, \alpha)=\widetilde{f}(\xi, \alpha) \chi(\alpha) \iint e^{i t\left\langle\gamma(\alpha)+u \gamma^{\prime}(\alpha), \xi\right\rangle} 2^{\ell} \widehat{\zeta}\left(2^{\ell} u\right) d u d t
$$

and therefore with $\chi_{0}(\alpha)=\chi(\alpha) \overline{\chi(\alpha)}$

$$
\begin{aligned}
\left\|\mathcal{B}_{\ell}^{*} f\right\|_{2}^{2}=(2 \pi)^{-3} \iiint \int \tilde{f}(\xi, t) \overline{\widetilde{f}\left(\xi, t^{\prime}\right)} & e^{i\left(t^{\prime}-t\right)\langle\gamma(\alpha), \xi\rangle} \\
& \times\left|\zeta\left(2^{-\ell}\left\langle\gamma^{\prime}(\alpha), \xi\right\rangle\right)\right|^{2} d t^{\prime} d t \chi_{0}(\alpha) d \alpha d \xi
\end{aligned}
$$

Let

$$
m_{\ell}(\sigma, \xi)=\int \chi_{0}(\alpha)\left|\zeta\left(2^{-\ell}\left\langle\gamma^{\prime}(\alpha), \xi\right\rangle\right)\right|^{2} e^{i \sigma\langle\gamma(\alpha), \xi\rangle} d \alpha .
$$

We shall show that

$$
\left|m_{\ell}(\sigma, \xi)\right| \lesssim 2^{-\ell} K_{\ell, \xi}(\sigma) \quad \text { where } \sup _{\ell, \xi}\left\|K_{\ell, \xi}\right\|_{L^{1}(\mathbb{R})}<\infty .
$$

The estimate (2.3) implies the asserted inequality since

$$
\left\|\mathcal{B}_{\ell}^{*} f\right\|_{2}^{2} \lesssim 2^{-\ell} \iiint\left|\widetilde{f}(\xi, t) \overline{\widetilde{f}\left(\xi, t^{\prime}\right)}\right| K_{\ell, \xi}\left(t-t^{\prime}\right) d t^{\prime} d t d \xi \lesssim 2^{-\ell}\|f\|_{2}^{2}
$$

Let

$$
\Sigma=\left\{\xi:\left\langle\gamma^{\prime}(s), \xi\right\rangle=\left\langle\gamma^{\prime \prime}(s), \xi\right\rangle=0 \quad \text { for some } s \in I_{0}\right\}
$$

which is the cone of binormal lines. Let $d_{\Sigma}(\xi)$ denote the distance of $\xi$ to $\Sigma$. (2.3) is a consequence of the following inequality expressing the behavior of $m_{\ell, \xi}$ in terms of $d_{\Sigma}(\xi)$. 
Lemma 2.3. For $\xi \in \mathbb{R}^{3}$

$$
\left|m_{\ell}(\sigma, \xi)\right| \lesssim 2^{-\ell} \frac{a_{\ell}(\xi)}{\left(1+a_{\ell}(\xi) \sigma\right)^{2}} \quad \text { where } a_{\ell}(\xi)=\frac{2^{2 \ell}}{\sqrt{|\xi|\left(2^{\ell}+d_{\Sigma}(\xi)\right)}} .
$$

Proof. Denote by $b=\left(\gamma^{\prime} \times \gamma^{\prime \prime}\right) /\left|\gamma^{\prime} \times \gamma^{\prime \prime}\right|$ the unit binormal vector (recall that we parametrized $\gamma$ by arclength). A conic neighborhood of the cone of binormal vectors is parametrized by $\psi(\rho, s, w)=\rho\left(b(s)+w \gamma^{\prime}(s)\right)$; here $\rho>0,|w|$ is small and $s$ belongs to a small neighborhood of supp $\chi$. One computes

$$
\left|\operatorname{det} \psi^{\prime}\right|=\rho^{2}|\tau-w \kappa|
$$

where $\kappa$ is the curvature and $\tau$ the torsion of the curve. This is a regular parametrization of the conic neighborhood of $\Sigma$; in this neighborhood $\rho w \approx d_{\Sigma}$. We chose $\varepsilon>0$ so that the set $\left\{\xi: d_{\sigma}(\xi) \leq 2 \varepsilon|\xi|\right\}$ is contained in this neighborhood.

In what follows choose the integer $k$ so that $2^{k}<|\xi| \leq 2^{k+1}$. Note that then $m_{\ell}(\sigma, \xi)=0$ if $\ell>k+C$ for suitable $C$ independent of $k$.

We shall first assume that $d_{\Sigma}(\xi) \geq \varepsilon|\xi|$. Note that for fixed $\xi$ the support of the cutoff $\zeta\left(2^{-\ell}\left\langle\gamma^{\prime}(\alpha), \xi\right\rangle\right)$ is contained in an interval of length $\approx 2^{\ell-k}$. This gives the bound

$$
\left|m_{\ell}(\sigma, \xi)\right| \lesssim 2^{\ell-k} \approx 2^{-\ell} a_{\ell}(\xi)
$$

which is sufficient if $|\sigma| \leq 2^{2 \ell-k}$. If $|\sigma|>2^{2 \ell-k}$ we integrate by parts twice and obtain the estimate

$$
\left|m_{\ell}(\sigma, \xi)\right| \lesssim 2^{\ell-k}\left(2^{2 \ell} \sigma\right)^{-2}
$$

and the two previous estimates yield the assertion if $d_{\Sigma}(\xi) \geq \varepsilon|\xi|$.

From now on we assume that $d_{\Sigma}(\xi) \leq \varepsilon|\xi|$; then $\xi=\rho\left(b(s)+w \gamma^{\prime}(s)\right)$ where $\rho \approx 2^{k},|w| \ll 1$ and that $\ell \ll k$. Observe that $\left\langle\gamma^{\prime \prime}(s), \xi\right\rangle=0$ and expand

$$
\left\langle\gamma^{\prime}(\alpha), \xi\right\rangle=\left\langle\gamma^{\prime}(s), \xi\right\rangle+(\alpha-s)^{2} G(s, \xi)
$$

where $G(\alpha, s, \xi)=\left\langle\xi, \int_{0}^{1}(1-\tau) \gamma^{\prime \prime \prime}(s+\tau(\alpha-s)) d \tau\right\rangle$; hence $|G(s, \xi)| \approx|\xi|$ and

$$
\left|\left\langle\gamma^{\prime \prime}(\alpha), \xi\right\rangle\right| \approx|\xi(\alpha-s)| \text {. }
$$

We first consider the case where $d_{\Sigma}(\xi) \leq C 2^{\ell}$; then $a_{\ell}(\xi) \approx 2^{(3 \ell-k) / 2}$. Assuming that $\left|\left\langle\gamma^{\prime}(\alpha), \xi\right\rangle\right| \approx 2^{\ell}$ we find that this can only occur on an interval of length $\lesssim 2^{(\ell-k) / 2}$ and that on this interval $\left|\left\langle\gamma^{\prime \prime}(\alpha), \xi\right\rangle\right| \lesssim 2^{(\ell+k) / 2}$. (To see this let $g(\alpha)=$ $\pm\left\langle\gamma^{\prime}(\alpha), \xi /|\xi|\right\rangle$ and expand $g(\alpha)=g(s)+g^{\prime \prime}(\tilde{\alpha})(\alpha-s)^{2} / 2$ with $\left|g^{\prime \prime}(\tilde{\alpha})\right| \approx 1$ and $|g(s)| \approx 2^{\ell-k}$.) We have to show that

$$
\left|m_{\ell}(\sigma, \xi)\right| \lesssim 2^{-\ell} \frac{2^{(3 \ell-k) / 2}}{\left(1+2^{(3 \ell-k) / 2} \sigma\right)^{2}}
$$

or, equivalently, that $\left|m_{\ell}(\sigma, \xi)\right| \lesssim 2^{(\ell-k) / 2}$ if $|\sigma| \leq 2^{(3 \ell-k) / 2}$ and $\left|m_{\ell}(\sigma, \xi)\right| \lesssim$ $2^{(k-5 \ell) / 2} \sigma^{-2}$ if $|\sigma|>2^{(3 \ell-k) / 2}$. The first estimate is immediate from the observation on the support, while the second is obtained using integration by parts, taking into account the bounds for the second and third derivatives.

Finally we consider the case $d_{\Sigma}(\xi) \approx 2^{\ell+n}$ where $0<n<k-l-c$. We change variables $\widetilde{\alpha}=(\alpha-s) \sqrt{|G(s, \xi /|\xi|)|}$ and use that the inequality

$$
\left|\frac{d_{\Sigma}(\xi)}{|\xi|}-\widetilde{\alpha}^{2}\right| \leq 2^{\ell-k+1}
$$


can only hold for $\widetilde{\alpha}$ on a set of measure $\leq C 2^{(\ell-k-n) / 2}$; the same restriction applies then to the set where $\left|\left\langle\gamma^{\prime}(\alpha), \xi\right\rangle\right| \approx 2^{\ell}$. On this set we have the estimate $\left|\left\langle\gamma^{\prime \prime}(\alpha), \xi\right\rangle\right| \lesssim 2^{(\ell+k+n) / 2}$. Integration by parts yields

$$
\left|m_{\ell}(\sigma, \xi)\right| \lesssim 2^{-\ell} \frac{2^{(3 \ell-k-n) / 2}}{\left(1+2^{(3 \ell-k-n) / 2} \sigma\right)^{2}}
$$

and since now $a_{\ell}(\xi) \approx 2^{3 \ell-k-n}$ this is the desired estimate.

Proof of Proposition 2.2. As in [15] we use the multilinear trick of [3]. Define

$$
\mathfrak{B}_{L}\left[f_{1}, \ldots, f_{6}\right]\left(x^{\prime}, t\right)=\prod_{i=1}^{6} \iint_{I \times \mathbb{R}} f_{i}\left(x^{\prime}+t \gamma(\alpha)+u \gamma^{\prime}(\alpha), \alpha\right) 2^{L} \eta\left(2^{L} u\right) d u \chi(\alpha) d \alpha ;
$$

then $\left\|B_{L} f\right\|_{6}^{6} \leq\left\|\mathfrak{B}_{L}[f, \ldots, f]\right\|_{1}$. We have to show that $\left\|\mathfrak{B}_{L}\left[f_{1}, \ldots, f_{6}\right]\right\|_{1}$ is dominated by $C 2^{2 L} \prod\left\|f_{i}\right\|_{3}^{6}$. By symmetry and interpolation (see [3]) this follows from

$$
\left\|\mathfrak{B}_{L}\left(f_{1}, \ldots, f_{6}\right)\right\|_{1} \lesssim 2^{2 L}\left\|f_{1}\right\|_{1} \prod_{i=2}^{6}\left\|f_{i}\right\|_{5,1}
$$

and it suffices to show this for $f_{1}$ replaced by a Dirac measure $\delta_{x^{0}}$ at $x^{0} \in \mathbb{R}^{4}$. By translation invariance in the $x^{\prime}$ variables we may assume that $x^{0}=(0,0,0, \beta)$ where $\beta \in \operatorname{supp} \chi$. Now

$$
\begin{aligned}
& \left\|\mathfrak{B}_{L}\left(\delta_{x^{0}}, f_{2}, \ldots, f_{6}\right)\right\|_{1} \\
& \leq \iint \prod_{i=2}^{6}\left[\iint_{I \times \mathbb{R}} \mid f_{i}\left(-t \gamma(\beta)-w \gamma^{\prime}(\beta)+t \gamma(\alpha)\right.\right. \\
& \left.\left.\quad+u \gamma^{\prime}(\alpha), \alpha\right) 2^{L} \eta\left(2^{L} u\right) \mid d u \chi(\alpha) d \alpha\right] 2^{L}\left|\eta\left(2^{L} w\right)\right| d w d t \\
& \leq \prod_{i=2}^{6}\left(\iint\left|B_{L} f_{i}\left(-t \gamma(\beta)-w \gamma^{\prime}(\beta), t\right)\right|^{5} 2^{L}\left|\eta\left(2^{L} w\right)\right| d w d t\right)^{1 / 5} .
\end{aligned}
$$

Define an operator $B_{L, \beta}$ mapping functions defined in $\mathbb{R}^{4}$ to functions defined in $\mathbb{R}^{2}$ by

$$
B_{L, \beta} f(t, w)=2^{L / 5}\left|\eta\left(2^{L} w\right)\right| B_{L} f\left(-t \gamma(\beta)-w \gamma^{\prime}(\beta), t\right) .
$$

In order to show the required estimate for $\mathfrak{B}_{L}$ we have to show that

$$
\left\|B_{L, \beta} f\right\|_{L^{5}\left(\mathbb{R}^{2}\right)} \leq C 2^{2 L / 5}\|f\|_{L^{5,1}\left(\mathbb{R}^{4}\right)}
$$

where $C$ does not depend on $\beta$. To prove (2.5) we use duality; let $g \in L^{5 / 4}\left(\mathbb{R}^{2}\right)$, so that $\|g\|_{5 / 4} \leq 1$. Then

$$
\begin{aligned}
& \left|\int B_{L, \beta} f(t, w) g(t, w) d t d w\right| \\
& \leq \iiint \int_{I \times \mathbb{R}}\left|f(\phi(t, \alpha, u, w)) 2^{L} \eta\left(2^{L} u\right)\right| d u|\chi(\alpha)| d \alpha|g(t, w)| d t 2^{L / 5}\left|\eta\left(2^{L} w\right)\right| d w
\end{aligned}
$$

where

$$
\phi(t, \alpha, u, w)=\left(-t \gamma(\beta)-w \gamma^{\prime}(\beta)+t \gamma(\alpha)+u \gamma^{\prime}(\alpha), \alpha\right) .
$$


One computes that

$$
\left|\operatorname{det}\left(\phi^{\prime}\right)\right|=\Delta(\beta) \frac{(\beta-\alpha)^{4}}{6}+O\left(|\beta-\alpha|^{5}\right)
$$

for $\alpha, \beta \in \operatorname{supp} \chi$ and since we assume that $\chi$ has small support we have

$$
\left|\operatorname{det}\left(\phi^{\prime}\right)\right| \approx|\alpha-\beta|^{4}:=J_{\beta}(\alpha)
$$

Since for fixed $\alpha$ the map $(t, u, w) \mapsto \phi(t, \alpha, u, w)$ is linear it follows that $\phi$ is injective in the regions $\alpha>\beta$ and $\alpha<\beta$ (here $\alpha, \beta \in \operatorname{supp} \chi$ ).

Now following [15] write

$$
\begin{aligned}
& \left|\int B_{L, \beta} f(t, w) g(t, w) d t d w\right| \\
& \quad \lesssim \iiint \int|f(\phi(t, \alpha, u, w))| G_{\beta}(t, w, u, \alpha) J_{\beta}(\alpha) d u d \alpha d t d w
\end{aligned}
$$

where

$$
G_{\beta}(t, w, u, \alpha)=2^{6 L / 5}\left|\frac{g(t, w)}{J_{\beta}(\alpha)} \chi\left(2^{L} u\right)\right| .
$$

Note that $\|f \circ \phi\|_{L^{5,1}\left(\mathbb{R}^{4}, J_{\beta} d m\right)} \leq 2\|f\|_{L^{5,1}\left(\mathbb{R}^{4}\right)}$ where $d m$ denotes Lebesgue measure in $\mathbb{R}^{4}$. Therefore the expression in (2.6) is dominated by

$$
C\|f\|_{L^{5,1}\left(\mathbb{R}^{4}\right)}\left\|G_{\beta}\right\|_{L^{5 / 4, \infty\left(\mathbb{R}^{4}, J_{\beta} d m\right)}}
$$

and, in order to finish the argument, one has to show that

$$
\left\|G_{\beta}\right\|_{L^{5 / 4, \infty}\left(\mathbb{R}^{4}, J_{\beta} d m\right)} \lesssim 2^{2 L / 5}\|g\|_{L^{5 / 4}\left(\mathbb{R}^{2}\right)} .
$$

To see (2.7) let $\chi_{L, 0}$ be the characteristic function of the interval $\left[-2^{-L}, 2^{-L}\right]$ and for $n=1,2, \ldots$ let $\chi_{L, n}(u)=1$ if $2^{-L+n-1} \leq|u| \leq 2^{-L+n}$ and $\chi_{L, n}(u)=0$ otherwise. Then $G_{\beta} \leq \sum_{n=0}^{\infty} G_{\beta, n}$ where

$$
G_{\beta, n}(t, w, u, \alpha)=C_{N} 2^{-n N} 2^{6 L / 5}\left|\frac{g(t, w)}{J_{\beta}(\alpha)}\right| \chi_{L, n}(u) .
$$

But

$$
\begin{aligned}
\int_{G_{\beta, n}>\lambda} & \left|J_{\beta}(\alpha)\right| d t d \alpha d u d w \\
& \lesssim 2^{-L+n+1} \iiint_{|\alpha-\beta| \leq\left(C_{N} 2^{-n N} 2^{6 L / 5}|g(t, w)| \lambda^{-1}\right)^{1 / 4}}|\alpha-\beta|^{4} d \alpha d t d w \\
& \lesssim 2^{-L+n+1} \iint\left(C_{N} 2^{-n N} 2^{6 L / 5}|g(t, w)| \lambda^{-1}\right)^{5 / 4} d t d w
\end{aligned}
$$

hence

$$
\left\|G_{\beta, n}\right\|_{L^{5 / 4, \infty}\left(\mathbb{R}^{4}, J_{\beta} d m\right)} \lesssim 2^{2 L / 5} 2^{-n(N-4 / 5)}\|g\|_{L^{5 / 4}\left(\mathbb{R}^{2}\right)}
$$

which implies (2.7). 


\section{INTEGRALS ALONG CURVES}

We sketch a proof showing that the operator defined by

$$
\mathcal{A} f(x)=\int_{-\infty}^{\infty} f\left(x-\Gamma_{4}(t)\right) d t
$$

maps $L^{5 / 2,1}\left(\mathbb{R}^{4}\right)$ to $L^{10 / 3, \infty}\left(\mathbb{R}^{4}\right)$. By duality it then maps $L^{10 / 7,1}\left(\mathbb{R}^{4}\right)$ to $L^{5 / 3, \infty}\left(\mathbb{R}^{4}\right)$. This implies the estimates asserted for $\mathcal{A}_{1}$ in Theorem 2.2 as well as $L^{p} \rightarrow L^{q}$ boundedness of $T_{0}$ in $(1.4)$, for $(1 / p, 1 / q) \in \mathcal{T}_{4} \backslash\left\{C_{4}, D_{4}\right\}$. The estimates for $\mathcal{A}_{\beta}$, $0<\beta<1$, will follow from these local estimates and Proposition 4.1 below.

The proof is quite close to the one in [15]; therefore we shall just sketch the argument. Define

$$
\mathcal{A}_{L} f(x)=\iint f\left(x-\Gamma_{4}(t)-u \Gamma_{4}^{\prime \prime}(t)\right) 2^{L} \eta\left(2^{L} u\right) d t d u .
$$

A modification of the multilinear argument in [15] yields that $\mathcal{A}_{L}$ is bounded from $L^{3}$ to $L^{6}$ with norm $O\left(2^{L / 3}\right)$. Arguing as in the proof of Theorem 1.1 we have to show that $\mathcal{A}_{\ell}-\mathcal{A}_{\ell+1}$ is bounded on $L^{2}$ with norm $O\left(2^{-\ell / 2}\right)$. This in turn follows from the estimate

$$
\begin{gathered}
\left|m_{\ell}(\xi)\right| \leq C 2^{-\ell / 2} \\
\text { where } \quad m_{\ell}(\xi)=\iint e^{i\left(\left\langle\xi, \Gamma(t)+u \Gamma^{\prime \prime}(t)\right\rangle\right)}\left(2^{\ell} \eta\left(2^{\ell} u\right)-2^{\ell+1} \eta\left(2^{\ell+1} u\right)\right) d t d u
\end{gathered}
$$

The proof of (3.2) is easier than the proof of the corresponding estimate in Proposition 2.1 since it can be deduced from known estimates for damped oscillatory integrals. Namely if

$$
\mathcal{J}_{\tau}(\xi)=\int_{-\infty}^{\infty} e^{i\langle\xi, \Gamma(t)\rangle}\left|\left\langle\xi, \Gamma^{\prime \prime}(t)\right\rangle\right|^{1 / 2+i \tau} d t
$$

then $\left|\mathcal{J}_{\tau}(\xi)\right| \lesssim(1+|\tau|)^{M}$, for some $M>0$, the bound is independent of $\xi$ (see [14]). Now let

$$
\theta(v)=\frac{\widehat{\eta}\left(e^{v}\right)-\widehat{\eta}\left(\frac{1}{2} e^{v}\right)}{e^{v / 2}}
$$

then $\theta \in C_{0}^{\infty}$ and a short computation using that $\eta$ is even yields

$$
\begin{aligned}
m_{\ell}(\xi) & =\int e^{i\langle\xi, \Gamma(t)\rangle}\left[\widehat{\eta}\left(2^{-\ell}\left|\left\langle\xi, \Gamma^{\prime \prime}(t)\right\rangle\right|\right)-\widehat{\eta}\left(2^{-\ell-1}\left|\left\langle\xi, \Gamma^{\prime \prime}(t)\right\rangle\right|\right)\right] d t \\
& =\int e^{i\langle\xi, \Gamma(t)\rangle} \theta\left(\log \left(2^{-\ell}\left|\left\langle\xi, \Gamma^{\prime \prime}(t)\right\rangle\right|\right)\right) 2^{-\ell / 2}\left|\left\langle\xi, \Gamma^{\prime \prime}(t)\right\rangle\right|^{1 / 2} d t \\
& =2^{-\ell / 2} \frac{1}{2 \pi} \int \widehat{\theta}(\tau) e^{-i \tau \ell \log 2} \mathcal{J}_{\tau}(\xi) d \tau
\end{aligned}
$$

Using the rapid decay of $\widehat{\theta}$ we see that the known estimate for $\mathcal{J}_{\tau}$ yields the bound (3.1).

Remark. We note that one could also work with the operator

$$
\widetilde{A_{L}} f(x)=\iint f\left(x-\Gamma_{4}(t)-u \Gamma_{4}^{\prime}(t)\right) 2^{L} \eta\left(2^{L} u\right) d t d u .
$$

Then $\widetilde{A}_{L}$ is bounded from $L^{3}$ to $L^{6}$ with norm $O\left(2^{2 L / 3}\right)$ and the operator $\widetilde{A}_{\ell}-\widetilde{A}_{\ell+1}$ is bounded on $L^{2}$ with norm $O\left(2^{-\ell}\right)$. 


\section{Estimates FOR FRACTIONAL INTEGRAL OPERATORS}

Suppose in $\mathbb{R}^{d}$ dilations $\delta_{t}$ are given by $\delta_{t} x=\exp (P \log t)$ where $P$ is a symmetric matrix so that all eigenvalues are positive. Let $\nu=\operatorname{trace}(P)$.

For a bounded Borel measure $\mu$ define the dilate of $\mu$ by

$$
\left\langle\mu_{k}, f\right\rangle=\int f\left(\delta_{2^{-k}} x\right) d \mu(x) .
$$

Given the result of the previous section, the following proposition implies Theorem 1.2 concerning the singular fractional integrals $\mathcal{A}_{\beta}$. The proof relies on an argument due to M. Christ [4] (a previous related result by Ricci and Stein [17] is applicable to a smaller set of exponents). In what follows we denote by $W F(\mu) \subset \mathbb{R}^{d} \times\left(\mathbb{R}^{d} \backslash\{0\}\right)$ the wavefront set of the measure $\mu$.

Proposition 4.1. Let $\mu$ be a positive finite Borel measure with compact support such that for all $x \in \mathbb{R}^{d}, \xi \neq 0$

$$
(x, \xi) \in W F(\mu) \Longrightarrow \xi \text { is not an eigenvector of } P \text {. }
$$

Let $\mu_{k}$ be defined by (4.1). Suppose $1<p<q<\infty, p \leq r$, and suppose the validity of the inequality

$$
\|\mu * f\|_{q, r} \leq A\|f\|_{p}
$$

Then the inequality

$$
\left\|\sum_{k=-N}^{N} 2^{-k \nu\left(\frac{1}{p}-\frac{1}{q}\right)} \mu_{k} * f\right\|_{q, r} \leq C(1+A)\|f\|_{p}
$$

holds with $C$ independent of $N$.

Proof. The proof relies on the observation that for $\xi \in W F(\mu)$ the $P$-dilates of a suitable conic neighborhood of $\xi$ have finite overlap. Without loss of generality we may assume that $P=\operatorname{diag}\left(a_{1}, \ldots, a_{n}\right)$, so that $\nu=\sum_{i=1}^{d} a_{i}$ and $\delta_{t} x=$ $\left(t^{a_{1}} x_{1}, \ldots, t^{a_{d}} x_{d}\right)$.

Note that if $\widehat{\chi} \in L^{1}$ and if $K$ is a convolution kernel so that $f \mapsto K * f$ is bounded from $L^{p}$ to $L^{q, r}$ (where $\left.1<p \leq q\right)$, then the operator $f \mapsto(\chi K) * f$ is also bounded from $L^{p}$ to $L^{q, r}$. This follows from $K * f=\int \widehat{\chi}(\xi) M_{\xi}\left[K *\left(M_{-\xi} f\right)\right] d \xi$, where $M_{\xi} f(x)=e^{i\langle x, \xi\rangle} f(x)$. Using nonnegative cutoff functions $\chi$ and the compactness of $\operatorname{supp} \mu$ we see that it is sufficient to prove that for every $x^{0} \in \mathbb{R}^{d}$ there is a neighborhood $U_{x^{0}}$ with compact closure such that the theorem holds for the measure $\chi \mu$, for all nonnegative $C^{\infty}$ functions $\chi$ supported in $U_{x^{0}}$.

We may assume that $W F(\mu) \neq \emptyset$, since otherwise $\mu \in C^{\infty}$. Indeed in this case $\sum_{k=-N}^{N} 2^{-k \nu / s} \mu_{k}$ is pointwise dominated by $c F_{s}$ where the constant $c$ does not depend on $N$ and

$$
F_{s}(x)=\left(\sum_{i=1}^{d}\left|x_{i}\right|^{1 / a_{i}}\right)^{-\nu+\nu / s}
$$

Now $F \in L^{s^{\prime}, \infty}$ and therefore the convolution operator $f \mapsto F_{s} * f$ is bounded from $L^{p} \rightarrow L^{q, p}$ if $1<p<q<\infty$ and $1 / s=1 / p-1 / q$.

Let $\mathcal{E}$ be the set of eigenvectors for $P$. Fix $x^{0}$; then we can find $U_{x_{0}}$ of $X^{0}$ and open conic neighborhoods $V, W$ of $\mathcal{E}$ such that the closure of $V \cap S^{d-1}$ is contained 
in $W \cap S^{d-1}$ and $\widehat{\chi \mu}(\xi) \leq C(1+|\xi|)^{-N}$ for $\xi \in W$, for all $\chi \in C_{0}^{\infty}\left(U_{x^{0}}\right)$. It suffices to prove the assertion for $\mu$ replaced by $\chi \mu$.

First observe that there is $\epsilon>0$ such that for every $u=\left(u_{1}, \ldots, u_{d}\right) \in S^{d-1} \backslash V$ we have

$$
\max \left\{\min \left(\left|u_{i}\right|,\left|u_{j}\right|\right): a_{i} \neq a_{j}\right\}>\epsilon .
$$

Therefore we may introduce a finite partition of unity $\left\{\eta_{m}\right\}_{m=1}^{M}$ of $S^{d-1} \backslash W$ where each $\eta_{m}$ is smooth on the sphere, extended as a smooth homogeneous function of degree 0 in $\mathbb{R}^{d} \backslash\{0\}$ so that $\sum_{m=1}^{M} \eta_{m}(\xi)=1$ for $\xi$ in an open neighborhood of $S^{d-1} \backslash W$ and the following two properties are satisfied.

There is $u^{m} \in S^{d-1} \backslash V$ such that the restriction of $\eta_{m}$ to the unit sphere is supported in $B_{m}=|\xi /| \xi\left|-u^{m}\right|<\varepsilon_{m}$, with $0<\varepsilon_{m}<\varepsilon / 4$, here $\varepsilon_{m}$ is chosen so that $|\xi /| \xi\left|-u^{m}\right|<2 \varepsilon_{m}$ implies $\xi \notin V$.

There are two indices $i(m), j(m)$ such that $a_{i(m)}>a_{j(m)}>0$ and $\left|u_{i(m)}^{m}\right| \geq \epsilon,\left|u_{j(m)}^{m}\right| \geq \epsilon$.

Suppose that $|\xi /| \xi\left|-u^{m}\right|<2 \varepsilon_{m}$. Since $2 \varepsilon_{m}<\varepsilon / 2$ we have

$$
\frac{\epsilon}{2(1+\varepsilon)} \leq \frac{\left|\xi_{i(m)}\right|}{\left|\xi_{j(m)}\right|} \leq 2 \frac{1+\varepsilon}{\epsilon} .
$$

Define $\widetilde{\eta}_{m}$ as a smooth homogeneous function of degree 0 so that $\widetilde{\eta}_{m}(\xi)=1$ for $0 \neq \xi \in \operatorname{supp} \eta_{m}$ and such that the restriction of $\widetilde{\eta}_{m}$ to the unit sphere is supported where $|\xi /| \xi\left|-u^{m}\right|<2 \varepsilon_{m}$.

Let $\Phi_{0}$ be smooth, compactly supported in $\{|\xi| \leq 2\}$ so that $\Phi_{0}(\xi)=1$ if $|\xi| \leq 1$. We define operators $P_{k}, S_{k}, L_{k}^{m}$ by

$$
\begin{aligned}
\widehat{P_{k} f} & =\Phi_{0}\left(\delta_{2^{-k}} \xi\right) \widehat{f}(\xi), \\
\widehat{L_{k}^{m} f} & =\eta_{m}\left(\delta_{2^{-k}} \xi\right) \widehat{f}(\xi), \\
\widehat{S_{k} f} & =\left(1-\Phi_{0}\left(\delta_{2^{-k}} \xi\right)\right)\left(1-\sum_{m=1}^{M} \eta_{m}\left(\delta_{2^{-k}} \xi\right)\right) \widehat{f}(\xi) ;
\end{aligned}
$$

then $I=P_{k}+S_{k}+\sum_{m}\left(I-P_{k}\right) L_{k}^{m}$. Define

$$
T_{k} f(x)=2^{-k \nu(1 / p-1 / q)} \mu_{k} * f .
$$

By assumption and scaling each operator $T_{k}$ is bounded from $L^{p} \rightarrow L^{q, r}$, with uniform operator norm $A$.

If $1 / s=1 / p-1 / q$, then the convolution kernels of $\sum_{k=-N}^{N} T_{k} P_{k}$ and $\sum_{k=-N}^{N} T_{k} S_{k}$ are pointwise bounded by $F_{s}$ in $(4.2)$, with $1 / s=1 / p-1 / q$; therefore those operators map $L^{p}$ to $L^{q, p}$ (and hence to $L^{q, r}$ since $r \geq p$ ).

We now estimate the main terms $\sum_{k=-N}^{N}\left(I-P_{k}\right) L_{k}^{m} T_{k}$. It is clear a priori that these operators map $L^{p}$ to $L^{q, p}$ with operator norm $O(N)$. In what follows let $B_{N}$ denote the $L^{p} \rightarrow L^{q, r}$ operator norm of the operator $\sum_{k=-N}^{N} T_{k}$. Following [4] we wish to show that $B_{N}=O(1)$.

Define $\widetilde{L}_{k}^{m}$ by $\widehat{\widetilde{L}_{k}^{m} f}=\widetilde{\eta}_{m}\left(\delta_{2^{-k}} \xi\right)$. In view of (4.4), (4.6) we may use the Marcinkiewicz multiplier theorem to see that $\sum_{k} b_{k} L_{k}^{m}$ and $\sum_{k} b_{k} \widetilde{L}_{k}^{m}$ are bounded operators on $L^{p}, 1<p<\infty$, uniformly for all sequences $b$ with $\|b\|_{\ell \infty} \leq 1$. Using 
a familiar argument involving Rademacher functions and real interpolation we see that the Littlewood-Paley inequalities

$$
\begin{aligned}
& \left\|\sum \widetilde{L}_{k}^{m} f_{k}\right\|_{p_{1}, p_{2}} \lesssim\left\|\left(\sum_{k}\left|f_{k}\right|^{2}\right)^{1 / 2}\right\|_{p_{1}, p_{2}}, \\
& \left\|\left(\sum_{k}\left|L_{k}^{m} f\right|^{2}\right)^{1 / 2}\right\|_{p_{1}, p_{2}} \lesssim\|f\|_{p_{1}, p_{2}}
\end{aligned}
$$

hold for $1<p_{1}<\infty, 1 \leq p_{2} \leq \infty$. By (4.7) and $\widetilde{L}_{k}^{m} L_{k}^{m}=L_{k}^{m}$ we have

$$
\left\|\sum_{k=-N}^{N}\left(I-P_{k}\right) L_{k}^{m} T_{k} f\right\|_{q, r} \lesssim\left\|\left(\sum_{k=-N}^{N}\left|\left(I-P_{k}\right) T_{k} L_{k}^{m} f\right|^{2}\right)^{1 / 2}\right\|_{q, r} .
$$

We shall use complex interpolation observing that

$$
\left.\left[L^{p, s}\left(A_{0}\right), L^{p, s}\left(A_{1}\right)\right]_{\theta}=L^{p, s}\left(\left[A_{0}, A_{1}\right)\right]_{\theta}\right)
$$

for a couple $\left(A_{0}, A_{1}\right)$ of compatible Banach-spaces. In particular

$$
\left[L^{p, s}\left(\ell^{p}\right), L^{p, s}\left(\ell^{\infty}\right)\right]_{\theta}=L^{p, s}\left(\ell^{2}\right) \text { if }(1-\theta) / p=1 / 2 .
$$

Since $p<q, p \leq r$, we may also use that

$$
\left\|\left(\sum_{k}\left|g_{k}\right|^{p}\right)^{1 / p}\right\|_{q, r} \lesssim\left(\sum_{k}\left\|g_{k}\right\|_{q, r}^{p}\right)^{1 / p}
$$

this is of course also true (with equality) if $p=q=r$. To see (4.11) observe that

$$
\begin{aligned}
\|h\|_{q, r} \approx & \left(\int_{0}^{\infty}\left[\alpha(\operatorname{meas}\{x:|h(x)|>\alpha\})^{1 / q}\right]^{r} \frac{d \alpha}{\alpha}\right)^{1 / r} \\
& =\left(\frac{1}{p} \int_{0}^{\infty}\left[\beta\left(\operatorname{meas}\left\{x:|h(x)|^{p}>\beta\right\}\right)^{p / q}\right]^{r / p} \frac{d \beta}{\beta}\right)^{1 / r}
\end{aligned}
$$

by a change of variable, hence $\|h\|_{q, r} \approx\left\||h|^{p}\right\|_{q / p, r / p}^{1 / p}$. Now $L^{q / p, r / p}$ is a normed space when $q / p>1$ and $r / p \geq 1$ (see [19]), and therefore (4.11) follows by applying (4.12) to $h=\left(\sum_{k}\left|g_{k}\right|^{p}\right)^{1 / p}$ and by applying the triangle inequality in $L^{q / p, r / p}$.

We use (4.11) with $g_{k}=\left(I-P_{k}\right) T_{k} f_{k}$ to obtain

$$
\begin{aligned}
& \left\|\left(\sum_{k=-N}^{N}\left|\left(I-P_{k}\right) T_{k} f_{k}\right|^{p}\right)^{1 / p}\right\|_{q, r} \lesssim\left(\sum_{k}\left\|\left(I-P_{k}\right) T_{k} f_{k}\right\|_{q, r}^{p}\right)^{1 / p} \\
& \quad \lesssim\left(\sum_{k}\left\|T_{k} f_{k}\right\|_{q, r}^{p}\right)^{1 / p} \lesssim A\left(\sum_{k}\left\|f_{k}\right\|_{p}^{p}\right)^{1 / p}=A\left\|\left(\sum_{k}\left|f_{k}\right|^{p}\right)^{1 / p}\right\|_{p}
\end{aligned}
$$

In view of the positivity and uniform $L^{q, r}$ boundedness of the operators and the $L^{q, r}$ boundedness of the Hardy-Littlewood maximal operator $\mathcal{M}$ we also have that

$$
\begin{aligned}
\left\|\sup _{-N \leq k \leq N}\left|\left(I-P_{k}\right) T_{k} f_{k}\right|\right\|_{q, r} & \lesssim\left\|\mathcal{M}\left(\sup _{-N \leq k \leq N}\left|T_{k} f_{k}\right|\right)\right\|_{q . r} \lesssim\left\|\sup _{-N \leq k \leq N}\left|T_{k} f_{k}\right|\right\|_{q, r} \\
& \lesssim\left\|\sum_{k=-N}^{N} T_{k}\left(\sup _{l \in \mathbb{Z}}\left|f_{l}\right|\right)\right\|_{q, r} \lesssim B_{N}\left\|\sup _{l \in \mathbb{Z}}\left|f_{l}\right|\right\|_{p} .
\end{aligned}
$$


We interpolate (4.13) and (4.14) using (4.9) and obtain

$$
\left\|\left(\sum_{k=-N}^{N}\left|\left(I-P_{k}\right) T_{k} f_{k}\right|^{2}\right)^{1 / 2}\right\|_{q, r} \lesssim A^{p / 2} B_{N}^{1-p / 2}\left\|\left(\sum_{k}\left|f_{k}\right|^{2}\right)^{1 / 2}\right\|_{p}
$$

We use this for $f_{k}=L_{k}^{m} f$ and apply the Littlewood-Paley inequalities (4.9), (4.8). These, together with (4.14) yield after summing the terms with $m=1, \ldots, M$,

$$
B_{N} \leq\left(C_{1}+C_{2} A^{p / 2} B_{N}^{1-p / 2}\right)
$$

and consequently

$$
B_{N} \leq C(1+A)
$$

\section{REFERENCES}

1. J. Bergh and J. Löfström, Interpolation spaces, Grundlehren der mathematischen Wissenschaften, 223, Springer Verlag, 1976. MR 58:2349

2. J. Bourgain, Estimations de certaines fonctions maximales, C. R. Acad. Sc. Paris, 301 (1985), 499-502. MR 87b:42023

3. M. Christ, On the restriction of the Fourier transform to curves: endpoint results and the degenerate case, Trans. Amer. Math. Soc. 287 (1985), 223-238. MR 87b:42018

4. _ Endpoint bounds for singular fractional integral operators, preprint, unpublished (1988).

5. I.M.Gelfand and M.I. Graev, Line complexes in the space $\mathbb{C}^{n}$, Func. Ann. Appl. 2 (1968), 219-229. MR 38:6522

6. A. Greenleaf and A. Seeger, Fourier integral operators with fold singularities, Jour. reine ang. Math. 455 (1994), 35-56. MR 95h:58130

7. __ Fourier integral operators with cusp singularities, Amer. J. Math. 120 (1998), 10771119. CMP 99:01

8. A. Greenleaf and G. Uhlmann, Nonlocal inversion formulas for the X-ray transform, Duke Math. J. 58 (1989), 205-240. MR 91b:58251

9. W. Littman, $L^{p}-L^{q}$-estimates for singular integral operators, Proc. Symp. Pure and Appl. Math. Amer. Math. Soc. 23 (1973), 479-481. MR 50:10909

10. D. McMichael, Damping oscillatory integrals with polynomial phases, Math. Scand. 73 (1993), 215-228. MR 95f: 42020

11. G. Mockenhaupt, A. Seeger and C.D. Sogge, Wave front sets, local smoothing and Bourgain's circular maximal theorem, Annals of Math. 136 (1992), 207-218. MR 93i:42009

12. A. Nagel, E. M. Stein and S. Wainger, Differentiation in lacunary directions, Proc. Nat. Acad. Sc. USA 75 (1978), 1060-1062. MR 57:6349

13. D. Oberlin, Convolution estimates for some measures on curves, Proc. Amer. Math. Soc. 99 (1987), 56-60. MR 88f: 42033

14. _ Oscillatory integrals with polynomial phase, Math. Scand. 69 (1991), 45-56. MR 93c: 41048

15. (1997), 1355-1361; II, Proc. Amer. Math. Soc. 127 (1999), 217-221. MR 97g:42009; CMP 99:01

16. __ An estimate for a restricted X-ray transform, preprint.

17. F. Ricci and E. M. Stein, Harmonic analysis on nilpotent groups and singular integrals III: Fractional integration along manifolds, J. Funct. Anal. 86 (1989), 360-389. MR 90m:22027

18. S. Secco, Fractional integration along homogeneous curves in $\mathbb{R}^{3}$, preprint. 
19. E. M. Stein and G. Weiss, Introduction to Fourier analysis on Euclidean spaces, Princeton Univ. Press, Princeton, N.J., 1971. MR 46:4102

20. R. Strichartz, Convolutions with kernels having singularities on the sphere, Trans. Amer. Math. Soc. 148 (1970), 461-471. MR 41:876

Department of Mathematics, University of Rochester, Rochester, New York 14627

E-mail address: allan@math.rochester.edu

Department of Mathematics, University of Wisconsin, Madison, Wisconsin 53706

E-mail address: seeger@math.wisc.edu

E-mail address: wainger@math.wisc.edu 